\title{
Praziquantel and Upper Gastrointestinal Bleeding in Hepatic Schistosomiasis: A Quasi-Experimental Study
}

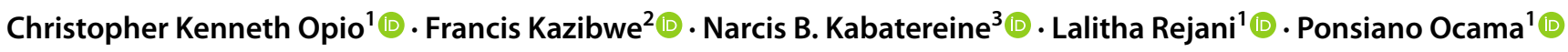

Accepted: 28 November 2020 / Published online: 26 December 2020

(c) The Author(s) 2020

\begin{abstract}
Background There is a general consensus that widespread use of praziquantel in populations where schistosomiasis is endemic prevents development of hepatic schistosomiasis and its complications. However, a few studies have reported discordant findings linking praziquantel to the occurrence of upper gastrointestinal bleeding (UGIB) in some patients with hepatic schistosomiasis and varices.

Objective We explored if there was any causal association between recent praziquantel use (rPZQ) and upper gastrointestinal bleeding in hepatic schistosomiasis in rural Africa.

Patients and Methods A quasi-experimental, retrospective case-controlled study was performed. It involved adult patients with past or acute UGIB, varices, periportal fibrosis, and/or cirrhosis. Cases had acute variceal bleeding while controls did not. The outcome was the frequency of lifetime episodes of UGIB and exposure was rPZQ (received praziquantel in the last 11 months from the date of enrollment). The data analysis included $2 \times 2$ tables, logistic regression, and propensity-score matching. Odds ratios (ORs), average treatment effects (ATEs), and their 95\% confidence intervals (CIs) were used for inference. Results Over 6 weeks, we enrolled 19 cases with 92 lifetime episodes of UGIB, and 66 controls with 192 lifetime episodes of UGIB. Cases were more likely to experience UGIB than controls following rPZQ (92\% vs. 62\%; OR 7.6; 95\% CI 3.4-17). Factors predictive of more lifetime episodes of UGIB at multivariable analysis included rPZQ (adjusted OR 13; 95\% CI 2.9-53), relative leukocytosis (adjusted OR 26; 95\% CI 7.6-89), large varices (adjusted OR 5.0; 95\% CI 1.7-15), a family member with hepatosplenic schistosomiasis (adjusted OR 19; 95\% CI 7.4-51), advanced periportal fibrosis (adjusted OR 8.0; 95\% CI 2.6-22), ascites (adjusted OR 14; 95\% CI 4.3-47), and jaundice (adjusted OR 32; 95\% CI 7.8-128). While the ATE following rPZQ among the treated was 0.40 (95\% CI 0.33-0.48).

Conclusions Our findings suggest the presence of a plausible causal association between recent praziquantel use and increased frequency of UGIB in our study population.
\end{abstract}

Supplementary Information The online version contains supplementary material available at https://doi.org/10.1007/s4080 1-020-00222-7.

Christopher Kenneth Opio

opiokc@gmail.com

Extended author information available on the last page of the article

\section{Key Points}

Our study found that one out of four persons with varices and hepatosplenic schistosomiasis and/or cirrhosis who recently used PZQ (within the last 11 months) developed acute variceal bleeding.

We also demonstrated a strong causal association between recent praziquantel use and the occurrence of more episodes of UGIB in our study population even after accounting for other potential contributary factors.

These findings are significant in that they validate what others observed and re-emphasize the need for cautious use of praziquantel among patients with hepatic schistosomiasis and/or liver cirrhosis. 


\section{Introduction}

Praziquantel (PZQ) is currently the most effective drug for the treatment of schistosomiasis. By 2018, it was estimated that over 235 million people worldwide received PZQ for schistosomiasis. This was mainly as preventive chemotherapy through mass drug administration (MDA) $[1,2]$. In hepatic schistosomiasis (HS) due to Schistosoma mansoni, PZQ was shown to be effective in preventing, decreasing, and sometimes even reversing periportal fibrosis. This was reported to occur within 12 months of treatment. PZQ has also been shown to decrease portal vein diameter and to reduce the prevalence of esophageal varices [3-8]. Predictors of successful treatment of periportal fibrosis following PZQ use include female gender, young age, mild-to-moderate periportal fibrosis, hepatitis B surface antigen-negative status, low $S$. mansoni re-infection rates, and genetics or family pedigree [9-14]. Whereas PZQ is considered very safe, over the last 15 years, a number of drug safety concerns have emerged $[2,7]$. Most of these have been highlighted in a number of reports from clinical studies, anthropological studies, and drug information leaflets [5, 15-21]. However, a few reports linking PZQ use to the occurrence of hematemesis or upper gastrointestinal bleeding (UGIB) in hepatic schistosomiasis seem to have been overlooked. One community study reported two to 30 out of 1000 persons experienced hematemesis as a side effect of PZQ [5]. One cohort described a high variceal UGIB rate and death rate among those with advanced schistosoma periportal fibrosis, and previous UGIB at 1 year, despite recent PZQ and endoscopic sclerotherapy [22]. A second cohort study found prior use of PZQ in patients with hepatic schistosomiasis (HS) was associated with greater odds of experiencing acute variceal UGIB [23]. A third cohort looked at the long-term effects of PZQ among patients with HS. They showed that acute variceal bleeding was more frequent among those with a history of UGIB than those without any lifetime history of bleeding [24]. These reports suggest the presence of a drug safety signal (information on a new or known adverse event that is potentially caused by a medicine and that warrants further investigation). Yet, no further evaluation of this unanticipated and bizarre drug safety signal has been undertaken [25].

We set out to investigate such phenomena in rural Africa were schistosomiasis is endemic, and mass drug administration of PZQ is frequent. Specifically, we explored if there was any causal association between PZQ use and occurrence of UGIB among patients with UGIB (past or present), varices, and HS that could not be explained by something else.

\section{Patients and Methods}

\subsection{Ethics Approval}

The Makerere University, College of Medicine, Institutional Research and Ethics Committee (\#REC, REF2011-244) and Uganda National Council for Science and Technology (\#HS1620) approved the study. Written informed consent was obtained from each study participant. The study was conducted in accordance with the ethical standards as laid down in the 1964 Declaration of Helsinki and its later amendments or comparable ethical standards.

\subsection{Study Design and Setting}

A quasi-experimental, retrospective case-controlled study design was performed. Participants were recruited and assessed at Pakwach Health Centre IV, a primary healthcare facility situated along the banks of the Albert Nile in rural northwestern Uganda. This area is a persistent hot spot for S. mansoni infection despite a reported annual mass drug administration coverage of $75 \%[26,27]$.

\subsection{Study Participants}

Eligible participants included all patients attending Pakwach Health Centre IV, aged 12 years and above, who were able to provide written informed consent/assent, and who reported experiencing one or more episode(s) of upper gastrointestinal bleeding during their lifetime. Upper gastrointestinal bleeding was defined as any history of hematemesis (vomiting blood), melena (passing black or charcoal-colored stool), or hematochezia (fresh blood in stool). Any participant who was pregnant, HIV positive (as per medical records), or had any contraindication to upper gastrointestinal endoscopy was excluded.

\subsection{Defining Clinical Outcome Assessments, Cases, and Controls}

The main outcome was the sum of the frequency of UGIB (past episodes of UGIB and/or acute variceal bleeding) experienced by either cases or controls. Cases included inpatients admitted for acute variceal bleeding and liver disease (periportal fibrosis and/or cirrhosis). Controls included outpatients with prior history of upper gastrointestinal bleeding, ultrasound evidence of liver disease (periportal fibrosis and/or cirrhosis), esophageal varices at endoscopy, and did not have any evidence of acute variceal bleeding during the study period. We chose controls as patients who previously bled because of their high risk of re-bleeding $[22,24]$. Cases 


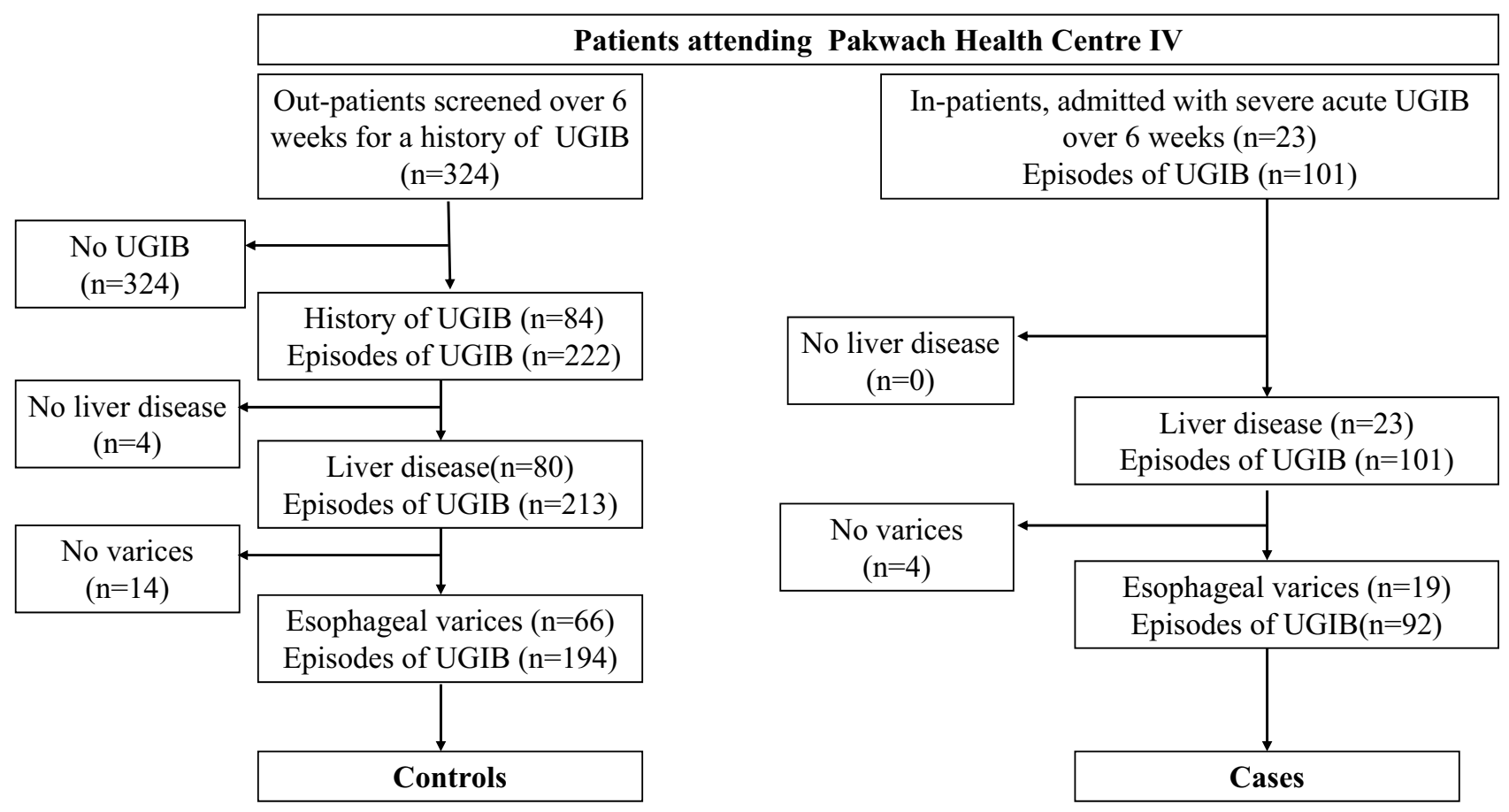

UGIB - Upper Gastrointestinal Bleeding

Fig. 1 Flow diagram showing the recruitment of cases and controls with the total number of episodes of upper gastrointestinal bleeding experience by participants at each stage of the recruitment process

and controls were recruited over the same period of 6 weeks. Figure 1 provides an overview of the recruitment process and outcome assessments.

\subsection{Study Procedures}

All cases and controls were subjected to the same study procedures. These included written informed consent, relevant medical history (which included history of previous PZQ use, period between last PZQ use and recruitment into the study, history of blood transfusion, past admissions, and others), a physical examination, routine clinical blood draws (for complete blood count, hepatitis B and C serologies), and urine for circulating cathodic antigen (CCA), stool for S. mansoni ova, trans-abdominal ultrasound (for diagnosis of periportal fibrosis due to schistosomiasis and cirrhosis), and diagnostic esophagogastroduodenoscopy for detection of esophageal varices. These clinical findings were recorded in a clinical report form, entered into a Microsoft Access database, and later exported to Stata version 15 for statistical analysis. The details of these procedures are described elsewhere [26, 28]. Potential confounders included gender, age, clinical jaundice, grade of periportal fibrosis, splenomegaly at ultrasound, ascites at ultrasound, grade of varices, prior blood transfusion, presence of hepatitis B infection, current infection with $S$. mansoni (urine circulating cathodic antigen test or presence of $S$. mansoni ova in a Kato Katz stool smear), enlarged portal vein diameter, and recent treatment with PZQ. The treatment variable was any participant treated with PZQ in the last 11 months (recent PZQ use), and treatment-independent variables or covariates included clinical jaundice, large esophageal varices, a family member diagnosed with hepatosplenic schistosomiasis, advanced periportal fibrosis, and total white cell count $\geq 3 \times 10^{9} / \mathrm{L}$.

\subsection{Statistical Analysis}

The initial analyses included $2 \times 2$ tables with frequencies, proportions, interval estimates, and $95 \%$ confidence intervals (CIs). Pearson's chi-square test, Fisher's exact test, and the rank-sum test were used for bivariate analysis. In addition, logistic regression with frequency weights of each covariate provided crude odds ratios (ORs), and their 95\% CIs. Multivariable logistic regression with frequency weights was performed by purposeful selection of covariates to control for confounding. Post-estimation of average marginal effects was then implemented for the best-fit model and this is summarized in a table format.

We estimated the treatment effects of PZQ (recent PZQ use) on the frequency of UGIB from our observational data 
by propensity-score matching in Stata using "teffects" command [29-31].

This was estimated using the derived logistic regression model, in which treatment status was regressed on the measured pretreatment characteristics. Matching was 1 to 1. To assume reasonable estimation of experimental-type causal effects, we checked for adequate balance of matching between cases and controls in Stata. This is presented graphically. Both average treatment effects on the study population and average treatment effects on the treated patients with their CIs were generated. Average treatment effects were presented as coefficients. These represented the absolute difference in average outcomes between controls and those recently treated with PZQ. For ease of interpretation of average treatment effects, we calculated the number needed to harm from the average treatment effects ( 1 divide by-average treatment effects). All tests were two-sided and a $p$-level significance value of $<0.05$ was considered. These results are presented in the text, tables, and as figures.

\section{Results}

\subsection{Frequency of Upper Gastrointestinal Bleeding (UGIB) and Time Since Last Treatment with PZQ}

During the months of July and August 2014, we studied 19 cases who experienced a total of 92 episodes of UGIB and 66 controls who experienced a total of 192 episodes of UGIB. Participants were grouped into five different period categories to determine a temporal relationship. These were defined by the period since a patient last used PZQ. Overall, the relative frequency of UGIB decreased with time since PZQ was last used.

Among the cases, 85 out of the 92 episodes of UGIB (93\%; 95\% CI 78-98) were experienced by those cases who received PZQ within the last 11 months. Among controls, 119 out of the 192 episodes of UGIB (62\%; 95\% CI 55-69) were experienced by those who received PZQ in the last 11 months. All cases received PZQ, while 13 (20\%) controls with 20 lifetimes episodes of UGIB had never received PZQ during their lifetime. Figure 2 summarizes these findings.

\subsection{Demographics and Relevant Medical History of Cases and Controls}

The median age (interquartile range) of cases and controls was 45 (11) years and 43 (14) years, respectively. The malefemale ratio among controls was five to eight and among cases it was eight to 11 . The lifetime frequencies of hematemesis, melena, and hematochezia among cases were 99\%, $86 \%$, and $83 \%$, respectively. While the lifetime frequencies of hematemesis, melena, and hematochezia among controls was $99 \%, 32 \%$, and 52\%, respectively. Recurrent UGIB (two or more episodes of UGIB) was more frequent among cases 15 (79\%) than controls 37 (56\%). Among cases (19 participants with a total of 92 episodes of UGIB), the median number
Fig. 2 Column chart describing the relative frequency episodes of upper gastrointestinal bleeding by praziquantel (PZQ) use over time among cases and controls. Relative frequency $=$ (Subgroup count /Total count) $\times 100$. PZQ use was assessed as used PZQ $<2$ months ago, used PZQ 2-11 months ago, used PZQ 12-59 months ago, used PZQ > 59 months ago, and never used PZQ
- Cases

Controls

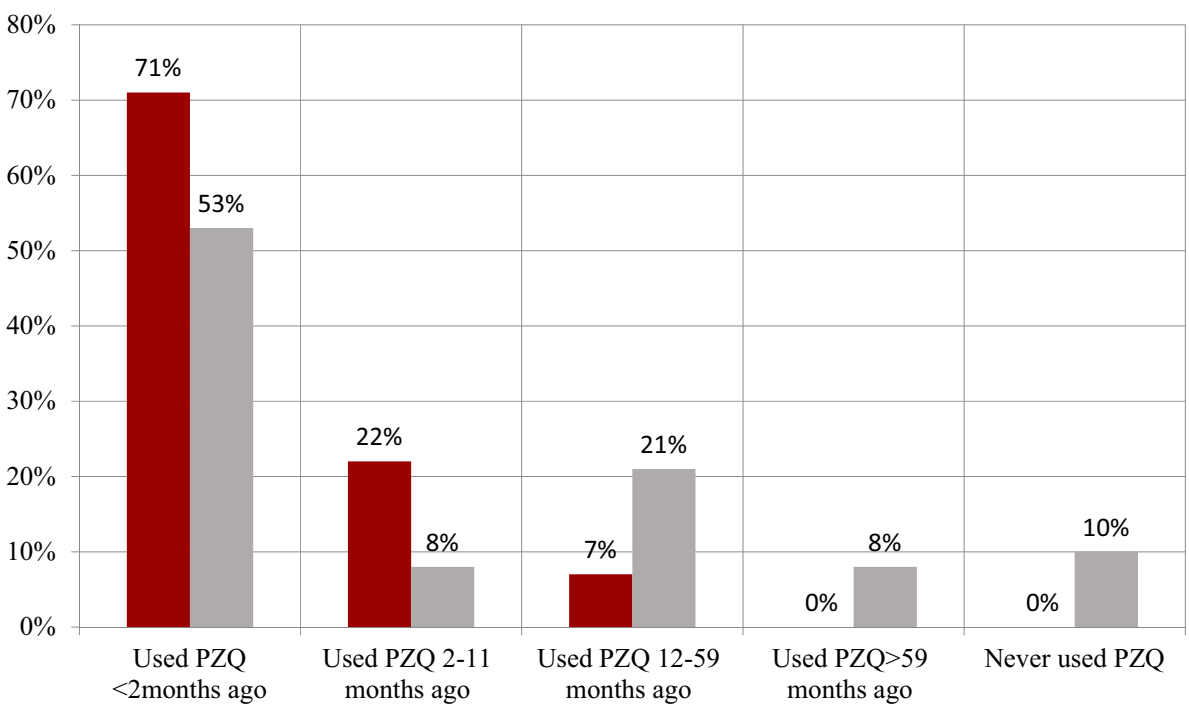

PZQ - Praziquantel , UGIB - upper gastrointestinal bleeding 
of episodes experience by each participant was three lifetime episodes. Among controls (66 participants with a total of 192 episodes of UGIB), the median number of episodes experience by each participant was two lifetime episodes. A comparison of these findings using the Mann-Whitney test or rank-sum test generated an exact $p$-value $=0.036$ (see supplementary tables in Online Supplementary Material, OSM). All cases $(100 \%)$ and almost all controls $(99 \%)$ reported a past admission for UGIB. Only one case and three controls used alcohol. Only one case and four controls had a positive urine CCA test. None of the cases or controls had ever been subject to diagnostic endoscopy nor had they received any specific treatment for variceal UGIB (propranolol, endoscopic band ligation or sclerotherapy, shunt surgery). Cases were treated medically for acute variceal bleeding. No control developed acute variceal UGIB during the study period and neither did any case re-bleed during the study period. All participants were started on propranolol after endoscopy for secondary prevention of variceal UGIB according to the current standard of care and no participant experienced recurrent UGIB during the 6-week study period [32].

\subsection{Contingency Table Analysis (Bi-Variable Analysis)}

Cases were 3.9 (95\% CI 1.1-14) times more likely to experience acute UGIB than controls if they received PZQ in the last 11 months (see OSM).

In addition, cases were 7.7 (95\% CI 3.4-17) times more likely to experience more lifetime episodes of UGIB than controls if they received PZQ in the last 11 months (recent PZQ use). This was also true for several factor variables including female gender (crude OR 3.4; 95\% CI 1.8-6.3), a family member diagnosed with hepatosplenic schistosomiasis (crude OR 6.2; 95\% CI 3.5-11), a relative leukocytosis of $\geq 3 \times 10^{9} / \mathrm{L}$ (crude OR 3.7 ; 95\% CI 2.0-6.5), large esophageal varices (crude OR 4.6; 95\% CI 2.5-8.4), advanced periportal fibrosis grades E or F (crude OR 4; 95\% CI 2.2-7.1), and jaundice (crude OR 25; 95\% CI 8.6-75) (Table 1).

\subsection{Multivariable Logistic Regression and Average Marginal Effects}

Factors predictive of more lifetime episodes of UGIB after multivariable analysis included "recent PZQ use" (adjusted OR 12; 95\% CI 2.9-53), relative leukocytosis (adjusted OR 26; 95\% CI 7.7-89), large varices (adjusted OR 5.0; 95\% CI 1.7-14.8), a family member with HS (adjusted OR 19; $95 \%$ CI 7.2-51), advanced periportal fibrosis (adjusted OR 7.6; 95\% CI 2.6-22), ascites (adjusted OR 14.2; 95\% CI 4.3-47), and jaundice (adjusted OR 31.5; 95\% CI 7.8-128) (Table 2). The C-statistic for this best model was $0.94, n=286$. The effects of the above factors on the probability of experiencing more episodes of UGIB were 22 percentage points increase for recent PZQ use, 29 percentage points increase for relative leukocytosis $\geq 3 \times 10^{9} / \mathrm{L}, 14$ percentage points increase for large varices, 26 percentage points increase for participants with a family history of hepatosplenic schistosomiasis, 18 percentage points increase for advanced periportal fibrosis (E, F), 23 percentage points increase for ascites, and 30 percentage points increase for jaundice. All these changes were statistically significant (Table 2).

\subsection{Estimated Average Treatment Effects and Number Needed to Harm}

The adjusted estimated treatment effects of PZQ on the frequency of UGIB and on acute variceal bleeding in our study population are summarized in Table 3 . The adjusted estimated treatment effect on the frequency of UGIB in the study population was 0.29 (95\% CI $0.23-0.35)$, while the average treatment effect among the treated was 0.41 (95\% CI $0.33-0.48)$. The number needed to harm was 3.3 (95\% CI 2.7-4.3) for the average treatment effects for the study population and the number needed to harm was 2.3 (95\% CI 2.1-2.9) for the average treatment effects on the treated cases (treated with PZQ in the last 11 months) (Table 3). The estimated average treatment effects of recent PZQ use on acute variceal bleeding and the number needed to harm following recent PZQ use are shown in Table 3.

\section{Discussion}

Our study looked at participants with a history of UGIB, ultrasound evidence of periportal fibrosis and/or cirrhosis, and esophageal varices. It showed participants who reported recent PZQ use had a greater probability of experiencing more episodes of UGIB than other participants (those who had never received PZQ and those who had not used PZQ recently). This probability was greatest among participants with advanced periportal fibrosis, large varices, ascites, jaundice, relative leukocytosis, and a family history of HS.

Chofle and others reported a history of previous PZQ use was associated with 4.4 times odds of developing acute variceal bleeding and a history of prior UGIB was associated with 2.7 times odds of developing acute variceal bleeding [23]. We found that recent PZQ use was associated with higher odds (OR 3.9; OSM) of experiencing acute variceal bleeding.

Richter and others found that recurrent variceal bleeding was more frequent among those with advanced fibrosis. From their study, most episodes of recurrent variceal bleeding occurred within the first 10 months of follow-up 
Table 1 The frequency of upper gastrointestinal bleeding (UGIB) among cases and controls by different factors and their crude odds ratios
Table 2 Multivariable regression and average marginal effects of various factors for prediction of upper gastrointestinal bleeding

\begin{tabular}{|c|c|c|c|}
\hline $\begin{array}{l}\text { Factor variables } \\
\text { Total UGIB episodes (\%) }\end{array}$ & Cases $(n=19)$ & Controls $(n=66)$ & \multirow[b]{3}{*}{ Crude odd ratios $(95 \% \mathrm{CI})$} \\
\hline \multirow[t]{2}{*}{ Total UGIB episodes (\%) } & $92(100)$ & $194(100)$ & \\
\hline & $\begin{array}{l}\text { UGIB episodes } \\
\text { among cases } n(\%)\end{array}$ & $\begin{array}{l}\text { UGIB episodes } \\
\text { among controls } n(\%)\end{array}$ & \\
\hline $\mathrm{PZQ} \leq 11$ months & $85(92)$ & $119(61)$ & $7.7(3.4-17)^{* * *}$ \\
\hline Age $\geq 40$ years & $79(86)$ & $152(78)$ & $1.7(0.9-3.3)$ \\
\hline Female & $77(84)$ & $117(60)$ & $3.4(1.8-6.3)^{* * *}$ \\
\hline Family member with HS & $73(79)$ & $19(10)$ & $6.2(3.5-11)^{* * *}$ \\
\hline Prior blood transfusion & $92(100)$ & $164(85)$ & - \\
\hline Spleen size $>12 \mathrm{~cm}$ & $92(100)$ & $178(92)$ & - \\
\hline Ascites at ultrasound & $23(25)$ & $40(21)$ & $1.3(0.71-2.3)$ \\
\hline Liver flap & 0 & $17(9)$ & - \\
\hline Clinical jaundice & $32(35)$ & $4(2)$ & $25(8.6-75)^{* * *}$ \\
\hline $\mathrm{WBC} \geq 3 \times 10^{9} / \mathrm{L}$ & $37(40)$ & $30(16)$ & $3.7(2.0-6.5)^{* * *}$ \\
\hline HBsAg positive & 0 & $10(5)$ & - \\
\hline Urine CCA positive & $2(2)$ & $5(3)$ & $0.4(0.09-1.9)$ \\
\hline Stool mansoni ova & 0 & $8(4)$ & - \\
\hline Liver fibrosis patterns $\mathrm{E}$ or $\mathrm{F}$ & $36(39)$ & $27(14)$ & $4.0(2.2-7.1)^{* * *}$ \\
\hline Large varices $(\mathrm{F} 3)$ & $76(83)$ & $99(51)$ & $4.6(2.5-8.4)^{* * *}$ \\
\hline
\end{tabular}

Percentages are rounded to the nearest whole number. The point estimates and their $95 \%$ CIs for crude odds ratios were obtained from logistic regression

$C I$ confidence interval, $P Z Q$ praziquantel, $W B C$ total white cell count, $C C A$ circulating cathodic antigen, $H B s A g$ hepatitis B surface antigen, $H S$ hepatic schistosomiasis

$* * * p$ value $<0.001$

\begin{tabular}{lcclc}
\hline$N=286$ & AOR (95\% CI) & $p$ value & $\begin{array}{l}\text { Average marginal } \\
\text { effects } \\
\text { Delta method dy/d } x \\
(95 \% \text { CI })\end{array}$ & $p$ value \\
\hline Family history of HS & & & $0.26(0.20-0.32)$ & 0.001 \\
Recent PZQ use ( $\leq 11$ months) & $13(2.9-53)$ & 0.001 & $0.22(0.10-0.34)$ & 0.001 \\
Large varices (F3 varices) & $5(1.7-15)$ & 0.004 & $0.14(0.05-0.23)$ & 0.003 \\
Advanced periportal fibrosis (pat- & $8(2.6-22)$ & 0.001 & $0.18(0.10-0.26)$ & 0.001 \\
$\quad$ terns E or F) & $26(7.6-89)$ & 0.001 & $0.29(0.20-0.38)$ & 0.001 \\
WBC $\geq 3 \times 10^{9} / \mathrm{L}$ & $14(4.3-47)$ & 0.001 & $0.22(0.15-0.32)$ & 0.001 \\
Ascites at ultrasound & $32(7.8-128)$ & 0.001 & $0.30(0.21-0.40)$ & 0.001 \\
Clinical jaundice & & & &
\end{tabular}

$A O R$ adjusted odds ratio, $P Z Q$ praziquantel, $F 3$ varices large varices, $H S$ hepatic schistosomiasis, $W B C$ total white cell count, $U G I B$ upper gastrointestinal bleeding
[22]. Advanced fibrosis was a predictor of UGIB in the same study. We found a significant association between the use of PZQ in 11 months and the occurrence of UGIB. We identified advanced periportal fibrosis, large varices, ascites, and jaundice as independent predictors of UGIB. Others have reported similar findings [14, 23, 33-35]. Our results also showed individuals with a close family member with HS were more likely to experience more episodes of UGIB. Though a family history of HS has not been directly associated with UGIB, progression of periportal fibrosis before and after PZQ use has been found to cluster in some families [9, 36-38]. From our study, participants who experienced more UGIB also had a relative leukocytosis. Leukocytosis is a frequent finding in UGIB and has been associated with poor outcomes $[39,40]$.

We explored the causal association between recent treatment with PZQ and UGIB by propensity-score matching [29]. Reasonable balance was achieved between cases and controls for the above factors. The effect of recent treatment with PZQ on the treated cases indicated a significant 
Table 3 Treatment effects of recent use of PZQ on occurrence acute variceal bleeding and lifetime frequency of UGIB

\begin{tabular}{|c|c|c|c|c|}
\hline Treatment effects & $n$ & Coef & $(95 \% \mathrm{CI}) p$ value & NNH $(95 \%$ CI $)$ \\
\hline $\begin{array}{l}\text { Estimated average treatment effect on lifetime frequency of UGIB in the whole } \\
\text { population }^{\mathrm{a}}\end{array}$ & 286 & 0.30 & $(0.23-0.36) p=0.001$ & $3.3(2.8-4.4)$ \\
\hline Estimated average treatment effect on lifetime frequency of UGIB in the treated ${ }^{\mathrm{b}}$ & 286 & 0.40 & $(0.33-0.48) p=0.001$ & $2.5(2.1-3.0)$ \\
\hline $\begin{array}{l}\text { Estimated average treatment effect on occurrence of acute variceal bleeding in the } \\
\text { study population }\end{array}$ & 85 & 0.17 & $(0.04-0.29) p=0.008$ & $5(3.5$ to 25$)$ \\
\hline $\begin{array}{l}\text { Estimated average treatment effect on occurrence of acute variceal bleeding in the } \\
\text { treated }^{\text {b }}\end{array}$ & 85 & 0.27 & $(0.13-0.40) p=0.001$ & $3.6(2.4-6.7)$ \\
\hline
\end{tabular}

UGIB upper gastrointestinal bleeding, $n=$ sample size, Coef coefficient (absolute difference in outcomes between controls and cases), NNH number needed to harm, $95 \%$ CI $95 \%$ confidence intervals

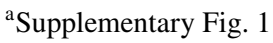

${ }^{\text {b}}$ Supplementary Fig. 2

absolute risk increase (average treatment effect) on the occurrence of UGIB with a number needed to harm of 2.5. Similar observations were made when we looked at the effects of recent treatment with PZQ on the treated regarding the occurrence of acute variceal bleeding. There was a significant absolute risk increase in occurrence of acute variceal bleeding with a number needed to harm of 3.6. These findings are similar to what Domingues and Coutinho reported from their 5-year cohort [24]. Their cohort described the long-term effects of PZQ chemotherapy on HS and found one out of three persons (number needed to harm of 3) developed UGIB after treatment with PZQ. From the number need to harm of 2.4-6.7, we estimated 149-417 of 1000 persons from our study population developed acute variceal bleeding following PZQ. A study from the Albert Nile basin found two to 30 out of 1000 persons $(0.9 \%)$ experienced hematemesis (a symptom of acute variceal bleeding) as a side effect of PZQ [5]. If we assume that about $10 \%$ of any population in an endemic region is likely to develop severe periportal fibrosis and hematemesis, a reasonable correction from our study would be 15-42 out of 1000 persons (149-417 divided by 10) [41]. This figure is similar to what Kabatereine and others reported [5].

We have no clear explanation for these unusual findings. Nonetheless, we postulate UGIB or hematemesis could be an indirect consequence of the primary action of PZQ on worms. We think PZQ causes the death of adult worms. These "dead adult worm lesions" may lead to intrahepatic thrombophlebitis, thrombosis, then a further increase in portal hypertension, and finally variceal bleeding. This phenomenon is probably limited to patients with a history of variceal UGIB who may not be able to compensate for changes in portal vein pressure resulting from "dead adult worm lesions" [42-45].

Our study has limitations We relied on patient reports to define PZQ use and the lifetime frequency of UGIB. This could have been subject to recall bias. For this reason, our results can be interpreted with caution. That said, patients' reports are valid and reliable outcome measures in care or in estimating recent medication use [15, 46-49]. In addition, patients' reports are a recognized and reliable source of drug safety signal in pharmacovigilance. Furthermore, our participants' reports were consistent with their clinical findings and published reports on HS, varices, and UGIB [46, 50-54]. Unintentionally, we could have excluded patients who died in the past, indicating our conclusions are only valid for those who survived. Although we used propensityscore matching to mitigate selection bias, we concede that we were only able to adjust for known confounding factors. Finally, this was a single-center study meaning our treatment effects could have been overstated.

\section{Conclusion}

Our study provides evidence suggesting a plausible causal association between recent PZQ use and increased frequency of UGIB in our study population. Our findings also offers another reason supporting the current manufacturers recommendation on the careful use of PZQ in patients with decompensated liver disease and prior UGIB [19-21]. These findings are important for ongoing drug pharmacovigilance and PZQ mass drug-administration programs. The study findings also underscore the pressing need for proper management of recurrent UGIB in this population through use of drugs, bands, and shunts [32].

Acknowledgements The study was funded through an educational research grant from the Programmatic Award: Medical Education for Services to All Ugandans (MESAU). http://www.fic.nih.gov/Grants/ Search/Pages/MEPI-R24TW008886.aspx. KReLL family for providing the endoscope tower. 


\section{Declarations}

Consent for publication The authors provide formal consent to publish this work.

Availability of data and materials Data are available and are provided as supplementary material.

Ethics approval The Makerere University, College of Medicine, Institutional Research and Ethics Committee (\#REC, REF2011-244) and Uganda National Council for Science and Technology (\#HS-1620) approved the study. Written informed consent was obtained from each study participant. The study was conducted in accordance with the ethical standards as laid down in the 1964 Declaration of Helsinki and its later amendments or comparable ethical standards.

Competing interests The authors declare that they have no competing interests.

Financial support The study was funded through an educational research grant from the Programmatic Award: Medical Education for Services to All Ugandans (MESAU). http://www.fic.nih.gov/Grants/ Search/Pages/MEPI-R24TW008886.aspx

Author contributions Protocol development: CKO, PO, FZ, LR. Data collection: CKO, PO, FZ, LR. Data analysis: CKO, PO, FZ, LR, NBK. Formulation of the manuscript: CKO, PO, FZ, LR, NBK. All authors read and approved the final manuscript.

Open Access This article is licensed under a Creative Commons Attribution-NonCommercial 4.0 International License, which permits any non-commercial use, sharing, adaptation, distribution and reproduction in any medium or format, as long as you give appropriate credit to the original author(s) and the source, provide a link to the Creative Commons licence, and indicate if changes were made. The images or other third party material in this article are included in the article's Creative Commons licence, unless indicated otherwise in a credit line to the material. If material is not included in the article's Creative Commons licence and your intended use is not permitted by statutory regulation or exceeds the permitted use, you will need to obtain permission directly from the copyright holder. To view a copy of this licence, visit http://creativecommons.org/licenses/by-nc/4.0/.

\section{References}

1. Vale N, Gouveia MJ, Rinaldi G, Brindley PJ, Gärtner F, Costa JMC da. Praziquantel for schistosomiasis: single-drug metabolism revisited, mode of action, and resistance. Antimicrob Agents Chemother [Internet]. 2017;61:e02582-16. http://aac.asm.org/ content/61/5/e02582-16. Accessed 22 July 2018.

2. Mutapi F, Maizels R, Fenwick A, Woolhouse M. Human schistosomiasis in the post mass drug administration era. Lancet Infect Dis. 2017; 17:e42-8.

3. Frenzel K, Grigull L, Odongo-Aginya E, Ndugwa CM, LoroniLakwo T, Schweigmann U, et al. Evidence for a long-term effect of a single dose of praziquantel on Schistosoma mansoni-induced hepatosplenic lesions in northern Uganda. Am J Trop Med Hyg. 1999;60:927-31.

4. Kheir MM. Effects of single-dose praziquantel on morbidity and mortality resulting from intestinal schistosomiasis. 2000;

5. Kabatereine NB, Tukahebwa E, Kazibwe F, Namwangye H, Zaramba S, Brooker S, et al. Progress towards countrywide control of schistosomiasis and soil-transmitted helminthiasis in Uganda. Trans R Soc Trop Med Hyg. 2006;100:208-15.

6. Danso-Appiah A, Olliaro PL, Donegan S, Sinclair D, Utzinger J. Drugs for treating Schistosoma mansoni infection. Cochrane Database Syst Rev. 2013;2013(2):CD000528. https://doi. org/10.1002/14651858.CD000528.pub2.

7. Zwang J, Olliaro PL. Clinical efficacy and tolerability of praziquantel for intestinal and urinary schistosomiasis - a meta-analysis of comparative and non-comparative clinical trials. PLoS Negl Trop Dis [Internet]. 2014; 8. https://www.ncbi.nlm.nih.gov/pmc/ articles/PMC4238982/. Accessed 11 Feb 2018.

8. Gameraddin MB. Sonographic assessment of the effect of praziquantel therapy on liver echotexture and diameters of the portal and splenic veins in patients with schistosomiasis. Radiol Infect Dis [Internet]. 2018;5:76-80.

9. Dessein A, Arnaud V, He H, Li J, Dessein H, Hou X, et al. Genetic analysis of human predisposition to hepatosplenic disease caused by schistosomes reveals the crucial role of connective tissue growth factor in rapid progression to severe hepatic fibrosis. Pathol Biol (Paris). 2013;61:3-10.

10. Rahoud SA, Mergani A, Khamis AH, Saeed OK, Mohamed-Ali Q, Dessein AJ, et al. Factors controlling the effect of praziquantel on liver fibrosis in Schistosoma mansoni-infected patients. FEMS Immunol Med Microbiol. 2010;58:106-12.

11. Berhe N, Myrvang B, Gundersen SG. Intensity of Schistosoma mansoni, hepatitis B, age, and sex predict levels of hepatic periportal thickening/fibrosis (PPT/F): a large-scale community-based study in Ethiopia. Am J Trop Med Hyg. 2007;77:1079-86.

12. Berhe N, Myrvang B, Gundersen SG. Reversibility of schistosomal periportal thickening/fibrosis after praziquantel therapy: a twenty-six month follow-up study in Ethiopia. Am J Trop Med Hyg. 2008;78:228-34.

13. Zwingenberger K, Richter J, Vergetti JS, Feldmeier H. Praziquantel in the treatment of hepatosplenic schistosomiasis: biochemical disease markers indicate deceleration of fibrogenesis and diminution of portal flow obstruction. Trans R Soc Trop Med Hyg. 1990;84:252-6.

14. Martins RD, Szejnfeld J, Lima FG, Ferrari AP. Endoscopic, ultrasonographic, and US-Doppler parameters as indicators of variceal bleeding in patients with schistosomiasis. Dig Dis Sci. 2000;45:1013-8.

15. Parker M, Allen T. Does mass drug administration for the integrated treatment of neglected tropical diseases really work? Assessing evidence for the control of schistosomiasis and soiltransmitted helminths in Uganda. Health Res Policy Syst [Internet]. 2011;9:3. https://doi.org/10.1186/1478-4505-9-3.

16. Parker M, Allen T, Hastings J. Resisting control of neglected tropical diseases: dilemmas in the mass treatment of schistosomiasis and soil-transmitted helminths in north-west Uganda. J Biosoc Sci. 2008;40:161-81

17. Muhumuza S, Olsen A, Katahoire A, Nuwaha F. Uptake of preventive treatment for intestinal schistosomiasis among school children in Jinja district, Uganda: a cross sectional study. PLoS One. 2013;8:e63438. https://doi.org/10.1371/journal.pone.0063438.

18. Hastings J. Rumours, riots and the rejection of mass drug administration for the treatment of schistosomiasis in Morogoro, Tanzania. J Biosoc Sci. 2016;48:S16-39.

19. el Guiniady MA, el Touny MA, Abdel-Bary MA, Abdel-Fatah SA, Metwally A. Clinical and pharmacokinetic study of praziquantel in Egyptian schistosomiasis patients with and without liver cell failure. Am J Trop Med Hyg. 1994;51:809-18.

20. Drugs@FDA: FDA Approved Drug Products [Internet]. https:// www.accessdata.fda.gov/scripts/cder/daf/index.cfm?event=overv iew.process\&ApplNo=018714. Accessed 25 May 2019. 
21. Biltricide (praziquantel) dose, indications, adverse effects, interactions... from PDR.net [Internet]. http://m.pdr.net/drug-summary/ Biltricide-praziquantel-2461. Accessed 22 July 2018.

22. Richter J, Correia Dacal AR, Vergetti Siqueira JG, Poggensee G, Mannsmann U, Deelder A, et al. Sonographic prediction of variceal bleeding in patients with liver fibrosis due to Schistosoma mansoni. Trop Med Int Health. 1998;3:728-35.

23. Chofle AA, Jaka H, Koy M, Smart LR, Kabangila R, Ewings FM, et al. Oesophageal varices, schistosomiasis, and mortality among patients admitted with haematemesis in Mwanza, Tanzania: a prospective cohort study. BMC Infect Dis. 2014;14:303.

24. Domingues ALC, Coutinho AD. Reduction of morbidity in hepatosplenic schistosomiasis mansoni after treatment with praziquantel: a long term study. Rev Soc Bras Med Trop. 1990;23:101-7.

25. Malikova MA. Practical applications of regulatory requirements for signal detection and communications in pharmacovigilance. Ther Adv Drug Saf [Internet]. 2020;11:2042098620909614. https ://doi.org/10.1177/2042098620909614.

26. Opio CK, Kazibwe F, Ocama P, Rejani L, Belousova EN, Ajal P. Profiling lifetime episodes of upper gastrointestinal bleeding among patients from rural Sub-Saharan Africa where schistosoma mansoni is endemic. Pan Afr Med J. 2016;24:296. https://doi. org/10.11604/pamj.2016.24.296.9755.

27. Exum NG, Kibira SPS, Ssenyonga R, Nobili J, Shannon AK, Ssempebwa JC, et al. The prevalence of schistosomiasis in Uganda: a nationally representative population estimate to inform control programs and water and sanitation interventions. PLoS Negl Trop Dis [Internet]. 2019;13:e0007617. https://doi. org/10.1371/journal.pntd.0007617.

28. Opio CK, Rejani L, Kazibwe F, Ocama P. The diagnostic accuracy of routine clinical findings for detection of esophageal varices in rural sub-Saharan Africa where schistosomiasis is endemic. Afr Health Sci. 2019;19:3225-34.

29. Rosenbaum PR, Rubin DB. The central role of the propensity score in observational studies for causal effects. Biometrika. 1983;70:41-55.

30. Suh H. Propensity Score Analysis Using teffects in STATA. University of Arizona. 2016

31. Abadie A, Imbens GW. Large sample properties of matching estimators for average treatment effects. Econometrica. 2006;74:235-67.

32. Opio CK, Garcia-Tsao G. Managing varices: drugs, bands, and shunts. Gastroenterol Clin. 2011;40:561-79.

33. Eltoum IA, Taha TE, Saad AM, Suliman SM, Bennett JL, Nash TE, et al. Predictors of upper gastrointestinal bleeding in patients with schistosomal periportal fibrosis. BJS. 1994;81:996-9.

34. Ibrahim SZ, Shah T, Arbab BM, Abdel Wahab O. Riskfactors for bleeding in patients with asymptomatic oesophageal varices secondary to schistosomal portal hypertension: a longitudinal hospital based study. Sudan Med J. 2009;45(1):35-41.

35. Mohammed SEA, Abdo AE, Mudawi HMY. Mortality and rebleeding following variceal haemorrhage in liver cirrhosis and periportal fibrosis. World J Hepatol. 2016;8:1336.

36. Tavares-Neto J, Prata A. Family occurrence of schistosomal hepatosplenomegaly and maternal effect. Rev Soc Bras Med Trop. 1989;22:13-8.

37. Ellis MK, McMANUS DP. Familial aggregation of human helminth infection in the Poyang lake area of China with a focus on genetic susceptibility to schistosomiasis japonica and associated markers of disease. Parasitology [Internet]. 2009;136:699-712. https://www.cambridge.org/core/journals/parasitology/artic le/familial-aggregation-of-human-helminth-infection-in-thepoyang-lake-area-of-china-with-a-focus-on-genetic-susceptibi lity-to-schistosomiasis-japonica-and-associated-markers-of-disea se/FF03351360F18C47004092AEBA0FB808. Accessed 18 Apr 2018.

38. Mahgoub MMO. Assessment of Praziquantel Effect and Some Genetic Factors on Prognosis of Periportal Fibrosis in Intestinal Schistosomiasis Patients in Gezira State. Sudan University of Science and Technology; 2015.

39. Chalasani N, Patel K, Clark WS, Wilcox CM. The prevalence and significance of leukocytosis in upper gastrointestinal bleeding. Am J Med Sci. 1998;315:233-6.

40. Moledina SM, Komba E. Risk factors for mortality among patients admitted with upper gastrointestinal bleeding at a tertiary hospital: a prospective cohort study. BMC Gastroenterol. 2017;17:165.

41. Conceição MJ, Borges-Pereira J, Coura JR. A thirty years follow-up study on Schistosomiasis mansoni in a community of Minas Gerais, Brazil. Memórias do Instituto Oswaldo Cruz. 2007;102:1007-9.

42. Johansen MV, Monrad J, Christensen N, Lindberg R. Experimental Schistosoma bovis infection in goats: pathological consequences of praziquantel treatment. J Comp Pathol. 1996;115:1-11.

43. Menezes H. Experimental intrahepatic portal embolism induced by adult Schistosoma mansoni. Am J Trop Med Hyg. 1963;12:741-4.

44. Tsutsumi H, Marcial-Rojas PA, Domingo J. Autopsy cases of schistosomiasis Mansoni in Puerto Rico. Kurume Med J. 1973;19:227-35.

45. de Andrade ZA. Schistosomiasis and liver fibrosis. Parasite Immunol. 2009;31:656-63.

46. Yang F, Tan X, Liu B, Yang C, Ni Z, Gao X, et al. Meta-analysis of the diagnostic efficiency of the questionnaires screening for schistosomiasis. Parasitol Res [Internet]. 2015;114:3509-19. https ://doi.org/10.1007/s00436-015-4579-8.

47. Rolfes L, van Hunsel F, Caster O, Taavola H, Taxis K, van Puijenbroek E. Does patient reporting lead to earlier detection of drug safety signals? A retrospective comparison of time to reporting between patients and healthcare professionals in a global database. Br J Clin Pharmacol. 2018;84:1514-24.

48. Inácio P, Cavaco A, Allan E, Airaksinen M. Key pharmacovigilance stakeholders' experiences of direct patient reporting of adverse drug reactions and their prospects of future development in the European Union. Public Health. 2018;155:119-28.

49. Drieling RL, LaCroix AZ, Beresford SAA, Boudreau DM, Kooperberg C, Heckbert SR. Validity of self-reported medication use compared with pharmacy records in a cohort of older women: findings from the women's health initiative. Am J Epidemiol [Internet]. 2016;184:233-8. Available from: https://www.ncbi. nlm.nih.gov/pmc/articles/PMC4967595/. Accessed 29 Jun 2019.

50. Rebouças G. Clinical aspects of hepatosplenic schistosomiasis: a contrast with cirrhosis. Yale J Biol Med. 1975;48:369.

51. Kabatereine NB, Ariho C, Christensen NO. Schistosoma mansoni in Pachwach, Nebbi District, Uganda, 40 years after Nelson. Trop Med Parasitol. 1992;43:162-6.

52. Lambertucci JR. Revisiting the concept of hepatosplenic schistosomiasis and its challenges using traditional and new tools. Rev Soc Bras Med Trop. 2014;47:130-6.

53. Ravera M, Reggiori A, Cocozza E, Ciantia F, Riccioni G. Clinical and endoscopic aspects of hepatosplenic schistosomiasis in Uganda. Eur J Gastroenterol Hepatol. 1996;8:693-7.

54. Ravera M, Reggiori A, Cocozza E, Andreata M, Ciantia F, Riccioni G. Clinical aspects and imaging patterns with ultrasonography and endoscopy in hepatosplenic schistosomiasis in Uganda. East Afr Med J. 1996;73:228-32. 


\section{Affiliations}

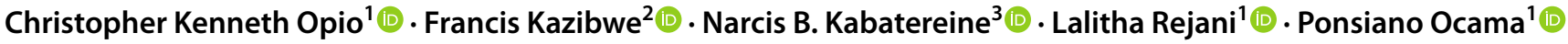

Francis Kazibwe

fkazibwe@gmail.com

Narcis B. Kabatereine

vcdmoh@gmail.com

Lalitha Rejani

rejaniopio@yahoo.com

Ponsiano Ocama

ponsiano.ocama@gmail.com
1 Makerere University College of Health Sciences, P. O. Box 7072, Kampala, Uganda

2 Public Health Department, Bishop Stuart University, P. O. Box 9, Mbarara, Uganda

3 Vector Control Division, Ministry of Health, P.O. Box 1661, Kampala, Uganda 